\title{
Comparative Experimental Analysis of Thermal Characteristics of Ytterbium-Doped Phosphosilicate and Aluminosilicate Fibers
}

\author{
Seungjong Lee ${ }^{1}$, Luis A. Vazquez-Zuniga ${ }^{1}$, Dongyoung Lee ${ }^{1}$, Hyuntai Kim ${ }^{1}$, \\ Jayanta K. Sahu ${ }^{2}$, and Yoonchan Jeong ${ }^{1 *}$ \\ ${ }^{1}$ Laser Engineering and Applications Laboratory, Department of Electrical and Computer Engineering, \\ Seoul National University, Seoul 151-744, Korea \\ ${ }^{2}$ Optoelectronics Research Centre, University of Southampton, Highfield, Southampton, SO17 1BJ, UK
}

(Received March 18, 2013 : revised April 1, 2013 : accepted April 5, 2013)

\begin{abstract}
We present a comparative experimental analysis of the thermal spectroscopic characteristics of a phosphosilicate (P)-based ytterbium-doped fiber (YDF) against an aluminosilicate (Al)-based YDF in the temperature range of 25 to $150^{\circ} \mathrm{C}$. We also characterize the fibers as gain media in a cladding-pumped amplifier configuration. While both fibers exhibit comparable trends in their thermal characteristics, there are noticeable distinctions in the fluorescence lifetime reduction rate and the spectral dependence of the transition cross-sections. The $\mathrm{P}$ - and $\mathrm{Al}$-based YDFs present thermal lifetime reduction rates of $0.012 \% /{ }^{\circ} \mathrm{C}$ and $0.026 \% /{ }^{\circ} \mathrm{C}$, respectively. In particular, in the spectral region at $\sim 940 \mathrm{~nm}$, the absorption cross-section of the P-based YDF undergoes significantly less thermal change compared to that of the Al-YDF. In the cladding-pumped amplifier configuration operating at a total gain of $10 \mathrm{~dB}$, the Al-based YDF generally performs betters than the P-based YDF in the temperature range of 25 to $75^{\circ} \mathrm{C}$. However, it is highlighted that in the high temperature range of over $75^{\circ} \mathrm{C}$, the latter shows a less gain reduction rate than the former, thereby yielding higher relative output power by $3.3 \%$ for a 1060 -nm signal, for example.
\end{abstract}

Keywords : Ytterbium-doped fibers, Ytterbium-doped fiber lasers, Phosphosilicate fibers, Aluminosilicate fibers

OCIS codes : (140.3510) Lasers, fiber; (140.3615) Lasers, ytterbium; (120.6810) Thermal effects; (300.6360) Spectroscopy, laser

\section{INTRODUCTION}

Ytterbium $(\mathrm{Yb})$ has been an outstanding choice as a doping material for rare-earth-doped fibers because of its exceptional spectroscopic properties, such as simple electronic states and small quantum defect, which in fact facilitate the power extraction from the gain medium with high efficiency [1-3]. However, thermal aspects with Yb-doped fibers (YDFs) cannot be simply overlooked if they operate in highpower regimes because their thermal properties are invariably linked to their long-term reliability as well as their overall efficiency [4-11]. For example, the efficiency of an YDF as a gain medium is dependent on the spectroscopy of $\mathrm{Yb}^{3+}$ ions that changes with temperature [5-7], and, in particular, photo-darkening (PD) effects are significantly affected with temperature [8-11]. Consequently, thermal characteristics, together with nonlinear scattering characteristics, can eventually be limiting factors to the performance of an YDF [12-13]. Thus, they have been intensively investigated from various prospects [4-11]. However, most of the related results have been limited to aluminosilicate (Al)-based YDFs [6,7] since they have predominantly been utilized in many high-power applications [1-3]. One of the reasons must be that they present higher transition cross-sections around the $1-\mu \mathrm{m}$ region compared to those of YDFs based on other host materials like phosphosilicate [14-16] as well as offering relatively low refractive core indices [1]. However, a main problem with Al-based YDFs is their vulnerability to PD [8-11, 16-18], particularly in fibers of high doping concentrations of $\mathrm{Yb}^{3+}$ ions [18]. Therefore, other types of YDFs have been considered as a replacement for Al-based YDFs

\footnotetext{
*Corresponding author: yoonchan@snu.ac.kr

Color versions of one or more of the figures in this paper are available online.
} 
$[15,19,20]$. Among the alternatives, phosphosilicate (P)-based YDFs have received a lot of attention since they can offer a superior property to Al-based YDFs in terms of PD resilience [19, 21]. Although there were some reports limited to the general spectroscopic characteristics of P-based YDFs $[14,16]$, to the best of our knowledge, there has been no detailed report on their thermal characteristics as gain fibers in high-power regimes. Here, building on our previous investigations [7], we present a comprehensive, comparative experimental analysis on the thermal characteristics of a P-based YDF against an Al-based YDF, including their spectroscopic properties, Stark energy levels, and gain characteristics in a cladding-pumped amplifier configuration. Finally, we discuss all the distinctive thermal characteristics between them.

\section{EXPERIMENTAL RESULT}

In addition to the Al-based YDF (YDF2/Al) previously studied in Ref. 7, a P-based YDF (YDF1/P) was newly fabricated by the modified chemical-vapor deposition and solution doping technique. Its core had a diameter of $6.1 \mu \mathrm{m}$ and a numerical aperture (NA) of 0.14. Its inner cladding was square-shaped with a side of $\sim 130 \mu \mathrm{m}$ and had a claddingabsorption rate of $\sim 1.8 \mathrm{~dB} / \mathrm{m}$ at $974 \mathrm{~nm}$. YDF2/Al had a core diameter of $6.6 \mu \mathrm{m}$ with an NA of 0.12 . Its inner cladding was hexagon-shaped with a diagonal of $\sim 130 \mu \mathrm{m}$ and had a cladding absorption rate of $\sim 2.4 \mathrm{~dB} / \mathrm{m}$ at 976 nm. In both fibers the inner cladding NA was higher than 0.45 . In addition, it should be noted that all the experimental measurements were done based on the methods and procedures introduced in our previous study unless stated otherwise (see Ref. 7 for details). We used a temperaturecontrolled oven to vary the fiber temperature from $25^{\circ} \mathrm{C}$ to $150^{\circ} \mathrm{C}$. It is noteworthy that the maximum temperature was limited by the thermal damage of the outer cladding material of the fiber that was made of low-index polymer [12].

Apart from other commonly used rare-earth ions, such as erbium $\left(\mathrm{Er}^{3+}\right)$, neodymium $\left(\mathrm{Nd}^{3+}\right)$, and thulium $\left(\mathrm{Tm}^{3+}\right)$ ions, the energy-level structure of $\mathrm{Yb}^{3+}$ ions has only two manifolds, the ground state ${ }^{2} F_{7 / 2}$ and the excited state ${ }^{2} F_{5 / 2}$ (see Table I) [6, 7, 14]. Each state is further split into Stark sublevels: The ground state ${ }^{2} F_{7 / 2}$ which has four sublevels (labeled from " $a$ " to " $d$ '), and the excited state ${ }^{2} F_{5 / 2}$ with three sublevels (labeled from " $e$ " to " $g$ "). The energy of the individual sublevel varies precisely depending on the composition of the host material [14]. In fact, the energy levels of $\mathrm{Yb}^{3+}$ ions and the transitions among the sublevels have close relationships with the transition (absorption and emission) cross-sections as well as the fluorescence lifetime $[22,23]$.

In order to determine the fluorescence lifetime, a $915-\mathrm{nm}$ laser diode driven in pulse mode was used to pump a short piece of $\mathrm{YDF} 1 / \mathrm{P}$, exciting the $\mathrm{Yb}^{3+}$ ions doped in the core. Fig. 1 shows the measured fluorescence lifetime

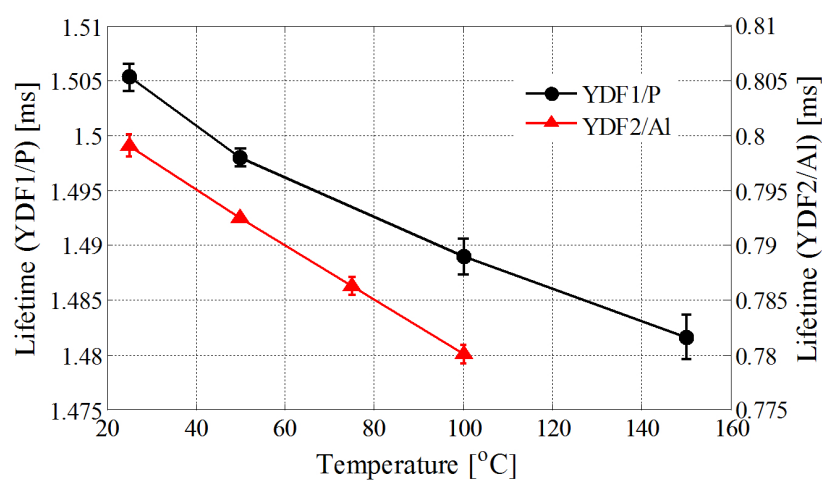

FIG. 1. Fluorescence lifetime of YDF1/P and YDF2/Al as a function of temperature. The error bars indicate the standard deviations of the measurements.

of YDF1/P together with that of YDF1/Al [7]. In particular, one can see that the measured fluorescence lifetime of YDF1/P was reduced from $1.505 \mathrm{~ms}$ at $25^{\circ} \mathrm{C}$ to $1.482 \mathrm{~ms}$ at $150^{\circ} \mathrm{C}$. The slight reduction of $1.53 \%$ in the fluorescence lifetime for the temperature difference $(\Delta \mathrm{T})$ of $125^{\circ} \mathrm{C}$ indicates that there was a non-negligible change in the probability distribution of population among the Stark sublevels [6]. It is also noteworthy that the fluorescence lifetime of YDF1/P is substantially longer than that of $\mathrm{YDF} 2 / \mathrm{Al}$, which exhibited a lifetime decrease from 0.799 to $0.780 \mathrm{~ms}$ with increasing temperature from 10 to $100^{\circ} \mathrm{C}$. This led to a $2.38 \%$ reduction for $\Delta \mathrm{T}=90^{\circ} \mathrm{C}$, which is relatively larger than that of YDF1/P.

As described above, the lifetime reduction along with temperature variation resulted from the change of the population distribution among the Stark sublevels of $\mathrm{Yb}^{3+}$ ions, which would subsequently give rise to modifications to their absorption and emissions cross-sections. In general, the absorption cross-section can readily be calculated via the following relation [7, 23]:

$$
\sigma_{a}(\lambda)=\frac{\alpha(\lambda)}{\Gamma(\lambda) N}
$$

where $\alpha(\lambda)$ is the absorption coefficient, $\Gamma(\lambda)$ the overlap factor between the doped area and the pump mode, $N$ the total number density, i.e., the concentration, of $\mathrm{Yb}^{3+}$ ions, and $\lambda$ the wavelength. The emission cross-section is also calculated via Füchtbauer-Ladenburg equation given by $[7$, 23, 24]

$$
\sigma_{e}(\lambda)=\frac{\lambda^{5}}{8 \pi c n^{2} \tau_{f}} \frac{\mathrm{I}(\lambda)}{\int \lambda \mathrm{I}(\lambda) d \lambda}
$$

where $I(\lambda)$ is the fluorescence intensity, and $c, n$, and $\tau_{f}$ denote the speed of light in vacuum, the refractive index of the medium, and the fluorescence lifetime, respectively. Thus, once the absorption coefficient and fluorescence 
intensity are measured, one can readily determine the transition cross-sections.

Figure 2 represents all the calculated absorption and emission cross-sections of YDF1/P and YDF2/Al based on our experimental measurements for different temperature conditions. The inset figures represent the fiber facet images of YDF1/P and $\mathrm{YDF} 2 / \mathrm{Al}$, respectively. It is noteworthy that the noticeable peaks and valleys of the cross-section curves are due to the different combinatorial transitions among the Stark sublevels. In addition, the specific energies of the individual Stark sublevels are summarized in Table 1, which

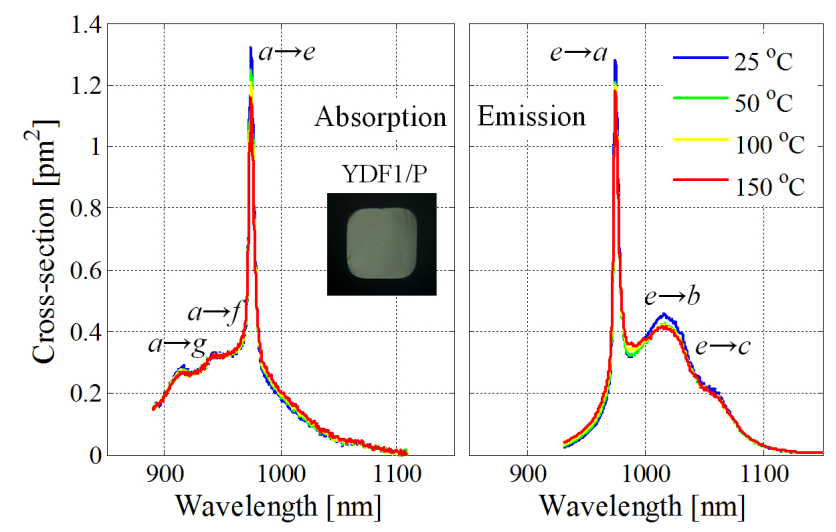

(a)

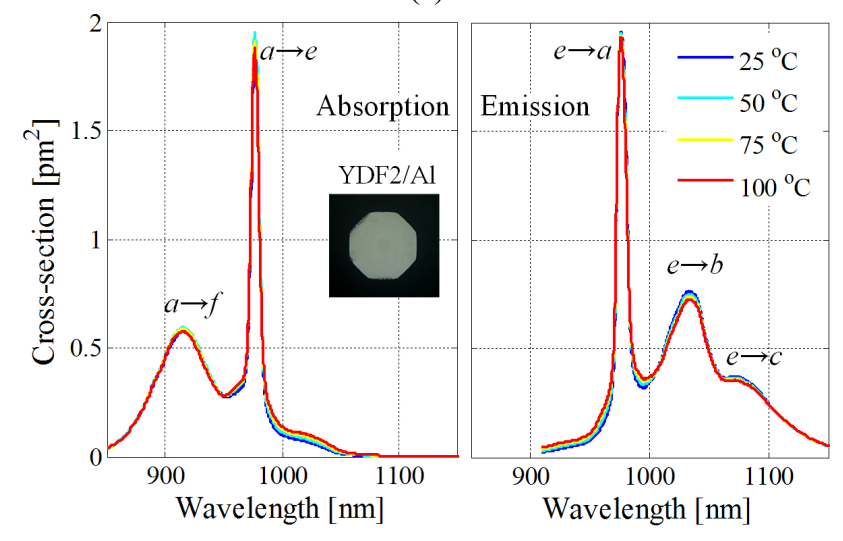

(b)

FIG. 2. Calculated absorption and emission cross-sections for (a) YDF1/P and (b) YDF2/Al for different temperature conditions. Inset: Fiber facet images of YDF1/P and YDF2/Al.

TABLE 1. The Stark energy-levels of YDF 1/P and YDF2/Al

\begin{tabular}{c|c|c|c}
\hline \multicolumn{2}{l|}{ Energy Levels } & YDF1/P $\left[\mathrm{cm}^{-1}\right]$ & YDF2/A1 $\left[\mathrm{cm}^{-1}\right]$ \\
\hline \multirow{3}{*}{${ }^{2} F_{5 / 2}$} & $g$ & 10915 & 11429 \\
\cline { 2 - 4 } & $f$ & 10617 & 10922 \\
\cline { 2 - 4 } & $e$ & 10262 & 10240 \\
\hline \multirow{4}{*}{${ }^{2} F_{7 / 2}$} & $d$ & 1087 & 1351 \\
\cline { 2 - 4 } & $c$ & 810 & 937 \\
\cline { 2 - 4 } & $b$ & 419 & 559 \\
\cline { 2 - 4 } & $a$ & 0 & 0 \\
\hline
\end{tabular}

were determined based on the emission and absorption spectra as well as their differential changes with temperature $[14,16]$. Actually, the estimated energies both for YDF1/P and YDF2/Al result in similar trends with other YDFs of the previous results $[14,16]$ while there are a bit of differences due to the detailed compositional variations.

In Fig. 2(a), the peaks of the absorption cross-section of YDF1/P, which are observed from $915 \mathrm{~nm}$ to $950 \mathrm{~nm}$, indicate the transitions of $a \rightarrow g$ and $a \rightarrow f$. The main peak observed at $\sim 974 \mathrm{~nm}$ corresponds to the transition of $a \rightarrow e$. The transitions from " $b$ " to upper sublevels represent the absorptions at longer wavelengths of $1030 \mathrm{~nm}$ to $1200 \mathrm{~nm}$. Regarding the emission cross-section of YDF1/P, the main peak located at $\sim 975 \mathrm{~nm}$ corresponds to the transition of $e \rightarrow a$. The transitions of $e \rightarrow b, e \rightarrow c$, and $e \rightarrow d$ correspond to the emissions peaks in the range of $1030 \mathrm{~nm}$ to $1200 \mathrm{~nm}$. The valleys in the cross-sections also represent other combinatorial transitions among the sublevels. In particular, the valley near $960 \mathrm{~nm}$ represents the transition of $f \rightarrow b$ and $b \rightarrow f$.

The transition cross-sections of $\mathrm{YDF} / \mathrm{Al}$ are also shown in Fig. 2(b). While they presented similar trends to those of YDF1/P to some extent, a clear noticeable difference between $\mathrm{YDF} 1 / \mathrm{P}$ and $\mathrm{YDF} 2 / \mathrm{Al}$ is the behavior in the region around $940 \mathrm{~nm}$. That is, the absorption cross-section of YDF1/P in that spectral band underwent significantly less thermal change compared to that of YDF2/Al. It should be noted that in that spectral region, $\mathrm{YDF} 1 / \mathrm{P}$ and $\mathrm{YDF} 2 / \mathrm{Al}$ form a plateau and a valley, respectively. As for the emission cross-sections, YDF1/P and YDF2/Al represent very similar behaviors, apart from the fact that the locations of the two main emission peaks in wavelengths above $1000 \mathrm{~nm}$ are substantially different: The two emission peaks of YDF1/P are located at much shorter wavelengths than those of $\mathrm{YDF} 2 / \mathrm{Al}$.

In addition, there are two significant, general trends in the changes of the transition cross-sections with temperature: One is that all the peaks related with the transitions to and from the lowest Stark sublevel, " $a$ ", decreases with temperature. The other is that the valleys related with the transitions between $f \rightarrow b$ and $b \rightarrow f$ become shallower with temperature. These are due mainly to the change in the population distribution of $\mathrm{Yb}^{3+}$ ions with temperature, which is primarily determined by the Boltzmann distribution law $[22,23]$. In other words, if temperature increases, the populations in the upper sublevels of ${ }^{2} F_{5 / 2}$ and ${ }^{2} F_{7 / 2}$ will grow, and this will subsequently reduce the populations in the lower sublevels. Therefore, this boosted upward migration of the population among the Stark sublevels results in the changes in the transition cross-sections in such ways as observed in Fig. 2. To quantify these consequences more precisely, we plot in Fig. 3 the deviations $\left(\Delta \bar{\sigma}_{a}\right.$ and $\left.\Delta \bar{\sigma}_{e}\right)$ of the transition cross-sections at 50 and $100^{\circ} \mathrm{C}$ against those at $25^{\circ} \mathrm{C}$, which are normalized by their maximum values at $25^{\circ} \mathrm{C}$ in order to make a fair comparison between them. 


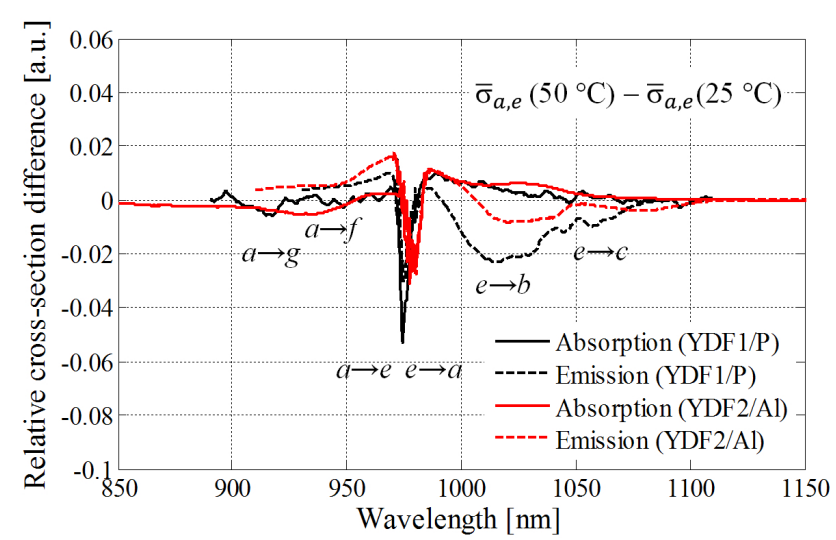

(a)

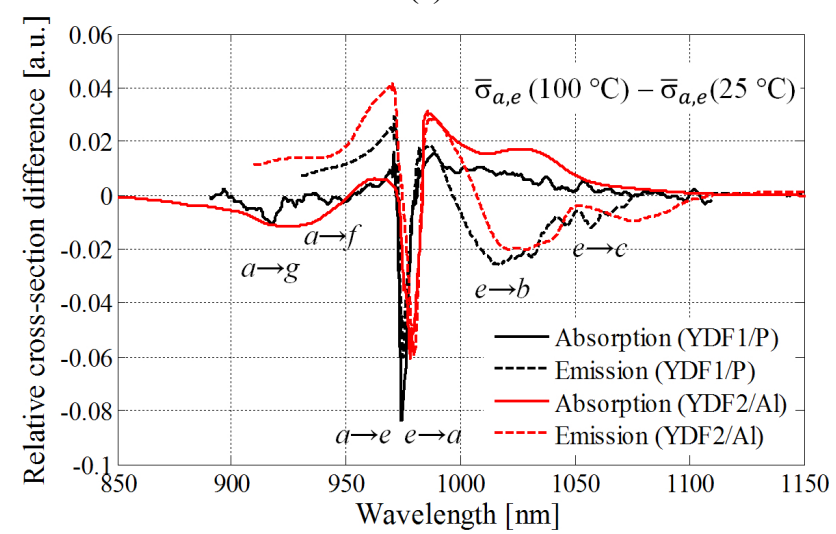

(b)

FIG. 3. Relative differences of the transition cross-sections at (a) 50 and (b) $100^{\circ} \mathrm{C}$ against those at $25^{\circ} \mathrm{C}$. The transition cross-sections are normalized to their maximum values at $25^{\circ} \mathrm{C}$.

As for $\Delta \bar{\sigma}_{a}$, there were clear indications of increasing quantities with temperature in the negative direction at around 920, 940, and $974 \mathrm{~nm}$ for $\mathrm{YDF} 1 / \mathrm{P}$, whereas at around 915 and $978 \mathrm{~nm}$ for YDF2/Al. These dips with negative quantities correspond to the transitions of $a \rightarrow g$, $a \rightarrow f$ and $a \rightarrow e$ for YDF1/P and those of $a \rightarrow f$ and $a \rightarrow$ $e$ for $\mathrm{YDF} 2 / \mathrm{Al}$. In particular, for $\mathrm{YDF} 1 / \mathrm{P}$, the thermal deviation in the absorption cross-section is relatively flat and small in the spectral range of $920 \mathrm{~nm}$ to $950 \mathrm{~nm}$, so that one can utilize this temperature insensitive spectral window for pumping, for example, pumping at $940 \mathrm{~nm}$. However, this is not the case with YDF2/Al: Its absorption cross-section at $915 \mathrm{~nm}$ is significantly reduced with temperature. The peak absorptions at $\sim 974$ and $\sim 975 \mathrm{~nm}$ for both cases exhibit relatively large variations with temperature. In addition, one can see that there were significant increases at the vicinity of $\sim 975 \mathrm{~nm}$ and even longer wavelengths in both YDF1/P and YDF2/Al. These correspond to the transitions started from non-ground levels, such as $b \rightarrow f$ and $c \rightarrow g$, which must increase with temperature according to the Boltzmann distribution law [22, 23]. In particular, in the wavelength range longer than $\sim 1020 \mathrm{~nm}, \mathrm{YDF} 1 / \mathrm{P}$ exhibits relatively smaller growth of the ground-state absorption in comparison with YDF2/Al.

As for $\Delta \bar{\sigma}_{e}$, the positive deviation is due mainly to the increase in the population in the upper Stark sublevels, such as, " $f$ " and " $g$ " levels whereas the negative deviation is due mainly to the decrease of the population in " $e$ " level. In particular, one can find an intriguing point here that in the wavelength range longer than $\sim 1020 \mathrm{~nm}$, the absolute quantity of $\Delta \bar{\sigma}_{e}$ for YDF1/P becomes comparable or relatively smaller than that of $\mathrm{YDF} 2 / \mathrm{Al}$ at the high temperature of $100^{\circ} \mathrm{C}$ whereas the former is significantly larger than the latter at the intermediate temperature of $50^{\circ} \mathrm{C}$. This feature is more significantly seen in the wavelength range above $\sim 1060 \mathrm{~nm}$. In fact, such changes in the spectroscopic properties will lead to gain reductions with temperature because the signals at those wavelengths will undergo more absorption but will acquire less emission. It should be noted that the gain is primarily determined by the transition cross-sections as well as the inversion ratio, fluorescence lifetime, etc [22, 23]. Thus, one can expect that YDF1/P should perform better than YDF2/Al in high temperature conditions because the thermal variations of the former's transition cross-sections tend to become less in comparison with those of the latter. Hence, we further investigated the temperature-dependent gain characteristics of YDF1/P and YDF2/Al when they were used as gain fibers in a cladding-pumped amplifier configuration in the following. In particular, the amplifier configuration was chosen rather than a laser configuration because its gain is directly related with the spectroscopic properties of the gain fiber, including the transition cross-sections as well as the fluorescence lifetime [22, 23].

We newly set up a tunable seed fiber laser and a cladding-pumped amplifier based on YDF1/P. This was in an identical format with our previous arrangement for YDF2/Al [7] except for the pumping wavelength: YDF1/P was pumped at $940 \mathrm{~nm}$ whereas $\mathrm{YDF} 2 / \mathrm{Al}$ was pumped at $915 \mathrm{~nm}$. The gain fiber of YDF1/P used in the amplifier was 23-m long and was placed in the temperature-controlled oven. It should be noted that in a free-running laser configuration both fibers presented $>70 \%$ of slope efficiencies with respect to absorbed pump power. Two different signal wavelengths of 1060 and $1080 \mathrm{~nm}$ were investigated for the temperature range of 25 to $100^{\circ} \mathrm{C}$. The seed power to the amplifier was fixed to $150 \mathrm{~mW}$ during the whole measurements, and then, the amplifier was pumped to yield $\sim 10-\mathrm{dB}$ gain, i.e., the output power of $\sim 1.5 \mathrm{~W}$, which was exactly the same condition as our previous arrangement for YDF2/Al [7]. It is noteworthy that we restricted the maximum temperature to $100^{\circ} \mathrm{C}$, which is well below the thermal damage threshold of the outer polymer material [12]. This was done in order to screen out any side effects due to the thermal degradation of the outer polymer material.

The gain characteristics of YDF1/P and YDF2/Al with temperature are shown in Fig. 4. It should be noted that 


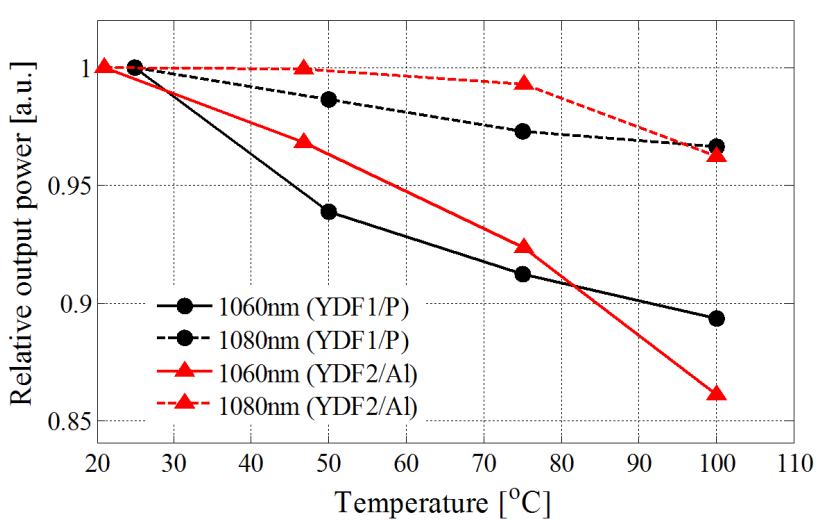

FIG. 4. Relative output powers of the cladding-pumped fiber amplifiers based on YDF1/P and YDF2/Al as a function of temperature. The output powers are normalized to their maximum values measured at $\sim 25^{\circ} \mathrm{C}$, which is $\sim 1.5 \mathrm{~W}$.

the output powers were normalized by their maximum values measured at $\sim 25^{\circ} \mathrm{C}$ in order to make a fair comparison between them. One can see a similar overall trend of decreasing output power with temperature, which is basically determined by the thermal changes of the spectroscopic properties of the fibers as discussed above. Since $\mathrm{Yb}^{3+}$ ions behave much like a quasi-three-level system owing to the Stark split of ${ }^{2} F_{7 / 2}$ which is not completely isolated from the ground state, they will suffer more ground-state absorptions if the signal wavelength becomes shorter. As temperature increases, the populations of " $b$ " and " $c$ " sublevels in ${ }^{2} F_{7 / 2}$ manifold will gradually grow, which will lead to more ground-state absorptions for 1060 and $1080 \mathrm{~nm}$ and less stimulated emissions for them as well. However, the strengths of such changes will be greater for $1060 \mathrm{~nm}$ than for $1080 \mathrm{~nm}$, as was verified in Fig. 3. This is why $1060 \mathrm{~nm}$ underwent more gain reduction than $1080 \mathrm{~nm}$. While both YDF1/P and YDF2/Al presented similar gain reduction trends, they made a clear distinction when the temperature was elevated above $\sim 80^{\circ} \mathrm{C}$. That is, the gain reduction rate of YDF1/P was significantly reduced down as the temperature increased whereas that of YDF2/Al further rapidly grew. This behavior can actually be expected if the temperature-dependent spectroscopic properties of YDF1/P and YDF2/Al are compared, based on the results shown in Fig. 3. In addition, one can also notice in Fig. 3, the absorption of $\mathrm{YDF} 2 / \mathrm{Al}$ at $915 \mathrm{~nm}$ (pump wavelength) was significantly reduced at the high temperature $\left(100^{\circ} \mathrm{C}\right)$ condition, which would subsequently give rise to additional gain reduction due to the decrease in the absorbed pump power. On the contrary, the absorption change of YDF1/P at $940 \mathrm{~nm}$ (pump wavelength) was nearly negligible even at the high temperature condition.

\section{CONCLUSION}

We have measured and analyzed thermal characteristics of a P-based YDF (YDF1/P) in comparison with an Al-based $\mathrm{YDF}$ (YDF2/Al), including their spectroscopic properties and gain characteristics in the temperature range of 25 to $100^{\circ} \mathrm{C}$. While both fibers exhibited similar and comparable trends in their thermal characteristics, we could see several noticeable distinctions in terms of the fluorescence lifetime reduction rate, the spectral dependence of the transition cross-sections, and the gain reduction rate. In particular, the fluorescence lifetime of YDF1/P was much longer $(\sim 1.5 \mathrm{~ms})$ than that of YDF2/Al ( $\sim 0.8 \mathrm{~ms})$, which implies that the lineshape broadening of the $\mathrm{Yb}^{3+}$ ions of the former must be smaller than that of the latter [22]. This can readily be seen in Fig. 3 or in Table 1. This, in fact, led to significantly less thermal lifetime reduction rate for $\mathrm{YDF} 1 / \mathrm{P}\left(0.012 \% /{ }^{\circ} \mathrm{C}\right)$ than that for $\mathrm{YDF} 2 / \mathrm{Al}\left(0.026 \% /{ }^{\circ} \mathrm{C}\right)$ [22]. Because of the narrower lineshape broadening of $\mathrm{YDF} 1 / \mathrm{P}$, the thermal changes of their transition cross-sections were more rapidly saturated in comparison with YDF2/Al. In addition, the absorption change of YDF1/P at $940 \mathrm{~nm}$ (pump wavelength) with temperature was nearly negligible. In result, YDF1/P exhibited better thermal resilience than $\mathrm{YDF} 2 / \mathrm{Al}$ in terms of gain reduction, particularly, at higher temperatures over $80^{\circ} \mathrm{C}$ when they were used as gain fibers in a cladding-pumped amplifier configuration. In this light the shortcomings of the quasithree-level nature of $\mathrm{Yb}^{3+}$ ions were better rectified in $\mathrm{YDF} 1 / \mathrm{P}$ than in $\mathrm{YDF} 2 / \mathrm{Al}$ in high-temperature conditions, which makes P-based YDFs attractive for stable, high-power operation, in particular, for operation at $1060 \mathrm{~nm}$. A P-based YDF has also demonstrated sufficiently high thermal damage resilience for high-power operation: In Ref. 21, a record power extraction of $1.17 \mathrm{~kW} / \mathrm{m}$ was successfully demonstrated, yielding the core temperature of well over $100^{\circ} \mathrm{C}[4,12]$. Therefore, we conclude that P-based YDFs must be a good replacement of Al-based YDFs, considering their thermally stable characteristics as well as their substantially high PD resilience [15,19,21]. Our comprehensive experimental data and comparative analysis on YDF1/P and YDF2/Al could be further utilized in rigorous modeling on YDFs.

\section{ACKNOWLEDGMENT}

This work was supported in part by the Ministry of Knowledge Economy (Project No. 10040429).

\section{REFERENCES}

1. Y. Jeong, J. K. Sahu, D. N. Payne, and J. Nilsson, "Ytterbium-doped large-core fiber laser with $1.36 \mathrm{~kW}$ continuouswave output power," Opt. Express 12, 6088-6092 (2004).

2. D. J. Richardson, J. Nilsson, and W. A. Clarkson, "High power fiber lasers: current status and future perspectives," J. Opt. Soc. Am. B 27, B63-B92 (2010).

3. J. Nilsson and D. N. Payne, "High-power fiber lasers," Science 332, 921-922 (2011). 
4. Y. Wang, C. Q. Xu, and H. Po, "Thermal effects in kilowatt fiber lasers," IEEE Photon. Technol. Lett. 16, 63-65 (2004).

5. T. C. Newell, P. Peterson, A. Gavrielides, and M. P. Sharma, "Temperature effects on the emission properties of $\mathrm{Yb}$-doped optical fibers," Opt. Commun. 273, 256-259 (2007).

6. X. Peng and L. Dong, "Temperature dependence of ytterbiumdoped fiber amplifiers," J. Opt. Soc. Am. B 25, 126-130 (2008).

7. L. A. Vazquez-Zuniga, S. Chung, and Y. Jeong, "Thermal characteristics of an ytterbium-doped fiber amplifier operating at 1060 and $1080 \mathrm{~nm}$, , Jpn. J. Appl. Phys. 49, 022502-1 022502-5 (2010).

8. M. J. Söderlund, J. Ponsoda, J. P. Koplow, and S. Honkanen, "Heat-induced darkening and spectral broadening in photodarkened ytterbium-doped fiber under thermal cycling," Opt. Express 17, 9940-9946 (2009).

9. S. Yoo, A. J. Boyland, R. J. Standish, and J. K. Sahu, "Measurement of photodarkening in Yb-doped aluminosilicate fibres at elevated temperature," Electron. Lett. 46, 233-234 (2010).

10. M. Leich, S. Jetschke, S. Unger, and J. Kirchhof, "Temperature influence on the photodarkening kinetics in Yb-doped silica fibers," J. Opt. Soc. Am. B 28, 65-68 (2011).

11. J. Ponsoda, C. G. Ye, J. P. Koplow, M. J. Söderlund, J. J. Koponen, and S. Honkanen. "Analysis of temperature dependence of photodarkening in ytterbium-doped fibers," Opt. Eng. 50, 111610 (2011).

12. Y. Jeong, S. Baek, P. Dupriez, J.-N. Maran, J. K. Sahu, J. Nilsson, and B. Lee, "Thermal characteristics of an endpumped high-power ytterbium-sensitized erbium-doped fiber laser under natural convection," Opt. Express 16, 19865-19871 (2008).

13. Y. Jeong, J. Nilsson, J. K. Sahu, D. N. Payne, R. Horley, L. M. B. Hickey, and P. W. Turner, "Erbium:ytterbium codoped large-core fiber laser with $297-\mathrm{W}$ continuous-wave output power," IEEE J. Select. Topics Quantum Electron. 13, 573-579 (2007).
14. M. A. Melkumov, I. A. Bufetov, K. S. Kravtsov, A. V. Shubin, and E. M. Dianov, "Lasing parameters of ytterbiumdoped fibres doped with $\mathrm{P}_{2} \mathrm{O}_{5}$ and $\mathrm{Al}_{2} \mathrm{O}_{3}$," Quantum Electron. 34, 843-848 (2004).

15. M. Engholm and L. Norin, "Preventing photodarkening in ytterbium-doped high power fiber lasers; correlation to the UV-transparency of the core glass," Opt. Express 16, 1260-1268 (2008).

16. S. Suzuki, H. A. McKay, X. Peng, L. Fu, and L. Dong, "Highly ytterbium-doped silica fibers with low photo-darkening," Opt. Express 17, 9924-9932 (2009).

17. J. J. Koponen, M. J. Söderlund, H. J. Hoffmann, and S. K. T. Tammela, "Measuring photodarkening from singlemode ytterbium doped silica fibers," Opt. Express 14, 11539-11544 (2006).

18. J. Jasapara, M. Andrejco, D. DiGiovanni, and R. Windeler, "Effect of heat and $\mathrm{H}_{2}$ gas on the photo-darkening of $\mathrm{Yb}^{+3}$ fibers," in Proc. CLEO/QELS (Long Beach, USA, May 2006), paper CTuQ5.

19. V. Shubin, M. V. Yashkov, M. A. Melkumov, S. A. Smirnov, I. A. Bufetov, and E. M. Dianov, in Proc. CLEO/IQEC (Munich, Germany, Jun. 2007), paper CJ3_1.

20. Y. W. Lee, S. Sinha, M. J. F. Digonnet, R. L. Byer, and S. Jiang, "Measurement of high photodarkening resistance in heavily $\mathrm{Yb}^{3+}$-doped phosphate fibres," Electron. Lett. 44, 14-16 (2008).

21. A. Codemard, A. Shirakawa, J. K. Sahu, S. Yoo, Y. Jeong, and J. Nilsson, "Thermal resilience of polymer-coated double-clad fiber," in Proc. CLEO/EQEC (Munich, Germany, Jun. 2009), paper CJ_P5.

22. W. Koechner, Solid-state Laser Engineering (Springer, London, UK, 1999).

23. E. Desurvire, Erbium-doped Fiber Amplifiers (Wiley, New York, USA, 1994).

24. X. L. Zou and H. Toratani, "Evaluation of spectroscopic properties of $\mathrm{Yb}^{3+}$-doped glasses," Phys. Rev. B 52, 1588915897 (1995). 